of muscular action by modifying the production of stimuli or changing their nature? That is, of course, the whole ground of controversy between the myogenist and the meurogenist. There are some recorded clinical experiences which appear to argue that the independence of the pro. perties ascribed to visceral muscle may be matter of legitimate doubt.

Jonesco, a surgeon of Bucharest, affirmed in 1897 that he had removed the entire chain of three sympathetic cervical ganglia with the intervening cord on both sides without ill result in a case of exophthalmic goitre, and, moreover, that he had never seen any evil consequences from this procedure. Some Parisian surgeons also report that they have cut the cord and removed the ganglia without a fatal result as a rule, although in one case, under the care of M. Faure, the patient suddenly died while under an anæsthetic after the cord and superior and inferior ganglia had been removed upon the right side. He suggests that the anresthetic (chloroform) had a share in the catastrophe. The experience of able surgeons in this country, however, indicates that fatal consequences may be directly attributable to that severance of the connexion between the heart and its nervous endowment which continental surgeons affirm they have effected without such a result. I have the permission of my friend, Mr. C. B. Lockwood, conscious of his strength, for recording his unfortunate experience of such a case, with notes of which he has supplied me.

The patient, a young woman, aged 19 years, suffering from exophthalmic goitre, with thyroid, heart and eye signs of two years' duration, was admitted into St. Bartholomew's Hospital on Jan. 18th, 1899, under the care of Dr. W. P. Herringham. A month later (on Feb. 18th), the patient having been anæsthetised with gas and ether, Mr. Lockwood operated upon the cervical sympathetic about 2 o'clock in the afternoon. The ganglia were not removed but the cervical cords were divided, that on the left completely, and a small portion excised; that on the right consisted of two cords, one of which was divided. The patient was removed to bed and quite recovered from the anæsthetic. The classical sigus of division of the sympathetic were well marked on the left side, the pupil of the left eye being contracted and the left half of the face flushed and occasionally sweating freely. The pulse and respiration were arcelerated and the temperature slightly raised from the commencement. The pulse became irregular and occasionally intermitted. As the day advanced these signs were more marked. The pupils also varied in size and at $11.20 \mathrm{P}$. Mr. they were equal and "pin-point." At 115 A.Mr. on the 19th the temperature was $105 \cdot 2^{\circ} \mathbf{F}$., the respirations were 60 , and the pulse was 200 . At 2.42 A.M. there were general convulsions. Both pupils were dilated and the left more than the right. At $2.45 \mathrm{~A} . \mathrm{N}$ the patient died, the temperature being $105-6^{\circ}$ and the left pupil being dilated and the right contracted.

A well-known provincial surgeon has also informed me that he divided the sympathetic cord on one side only and with fatal consequences in a case of exophthalmic goitre. It is difficult to reconcile these results with the experience of continental surgeons. Mr. Lockwood had some dificulty when operating on the chain in the right side of the neck in his case in determining in a leash of nerve cords which was the sympathetic, but the necropsy proved that he had completely divided the left and partially the right. Mr. F. F. Burghard of King's College Hospital informs me that he has on more than one occasion removed both the superior ganglia of the cervical sympathetic without fatal consequences, which is valuable clinical confirmation of the physiological conclusion from experiment that the chief cardiac outflow of visceral sympathetic nerves is through the inferior cervical ganglia without its previously traversing the superior ganglia.

Without wishing to be unduly sceptical one feels disposed to question whether there has not been some error as to complete division of the sympathetic in the cases reported to have suffered little inconvenience from the procedure. That an important nerve such as the pneumogastric may occasionally be divided on one side without fatal results is admitted, and the same is probably true of the sympathetic, but a complete division of the nerve on both sides, both as regards the pneumogastric and the sympathetic when the inferior cervical ganglion is involved, must on physiological grounds be scarcely compatible with life. Whatever view, therefore, may be taken of the essential nature of muscular and nervous action, it is manifest that their relation is so intimate in man as to render impossible persistent organic action when their union is severed, and we return to the position at which we commenced-namely, the tripartite unity of the hæmic, muscular, and nervous factors in cardiac action, which I imagine we all acknowledge to be essential to sustained organic activity.

So far, we have been concerned with a consideration of the essential or intrinsic causes of cardiac motion and therefore also with the causes of disorder in such function. Some reference may now be made to more accidental or extrinsic causes, for these also have an important bearing on treatment and, like all extrinsic or accidental circumstances, are at times more easy of correction than the essential causes of disordered cardiac action. Such extrinsic causes are mechanical and in their management frequently require the coöperation of the physician with the surgeon. They concern the blood-weight or distribution as distinct from the blood-supply to the cardiac muscle, the valvular mechanism to secure a due distribution of the blood, and space enough without impediment for the organ to contract and dilate and to receive and project its contents-in short, the causes which affect the working of the heart as distinct from those which underlie its action. The mechanical factors frequently dominate the situation, when, as so frequently happens, in addition to cardiac and intracardiac causes of difficult circulation, the movements of the organ are rendered more difficult by extracardiac adhesions to neigkbouring parts, or by notable impediments to full respiration dne to effusions of fluid or the presence of abnormal solids in the thoracic or abdominal cavities. The modification or, when possible, the removal of such aggravating mechanical factors then becomes a paramount necessity. It is manifest therefore that when the physician fails to restore an easy balance between the intrinsic factors in a labouring circulation by the indirect influence of the potent agents so frequently successful in his hands he must seek in some cases the coöperation of the surgeon in the modification or removal of such extrinsic factors as are amenable to bis art.

\section{ON THE TREATMENT OF FRACTURES IN THE VICINITY OF JOINTS.}

By W. ARBUTHNOT LANE, M.S. LoND., F.R.C.S. ENG., SURGEON TO GUY'S HOSPITAL AND SENIOR SURGEON TO THE HOSPITAD FUR SICK CHILDREN, GREAT ORMIOND-STREET, LONDON, W.C.

I Do not propose to say anything about the diagnosis of fractures, since it has resolved itself so largely into a question of obtaining perfect $x$ ray photographs. I will satisfy myself by urging the importance of obtaining photographs in several planes, since it is only in this way that the nature of the fracture can be accurately diagnosed. The x ray photograph also furnishes us with a ready means of determining the manner in which we have done our work, whether we have attempted to replace the fragments in accurate apposition by manipulation or by operation. In either case the part should inmediately after the operation be photographed in several planes, so that any defect may be effectually dealt with as soon as possible.

The fractures about the ankle-joint come under two distinct groups. First we have those produced by excessive abduction, which are called Pott's fractures and which consist in a spiral or torsion fracture of the lower portion of the fibula with or without rupture of the internal lateral ligament, or of transverse fracture of a portion of the internal malleolus with or without a vertical fracture of the posterior and outer part of the lower end of the tibia which is attached by ligament to the fibula. Secondly we have those produced by a fall on the foot in a position of considerable adduction or of activity. In these the inner malleolus with a portion of the tibia are broken off by a vertical fracture which usually traverses the tibia at the junction of the malleolus with the lower surface of the tibia. The malleolus with the portion of the shaft is displaced upwards upon the rest of the tibia. The fibula is broken transversely immediately above the attachment to the lower end of the tibia. The fracture is of the nature of a scrunch and the fragments may be much comminuted. Fig. 1 represents such a fracture taken from before backwards. This man to avoid being crushed jumped down into a ship's hold. The inner malleolus with a portion of the tibia was displaced upwards, 
while the fibular fracture was much comminuted. The tibial fragment was replaced in position and was retained by means of a steel plate fixed by two screws, a long one traversing the lower end of the tibia and a short one the inner malleolus. It did not appear necessary to do anything to

\section{FIG. 1.}

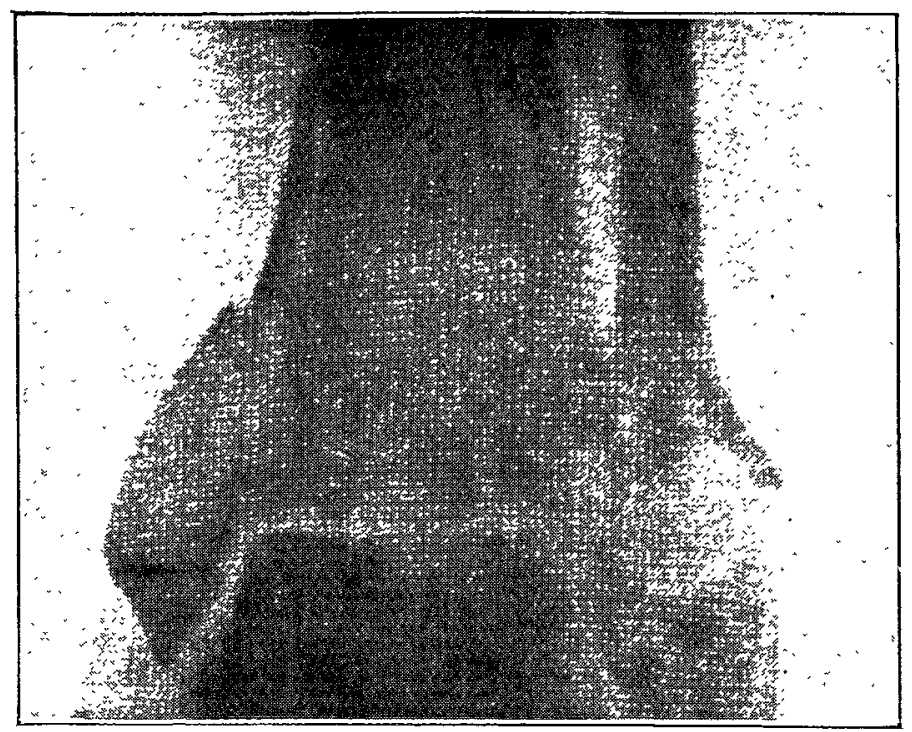

Represents an "adduction fracture" of the ankle taken from before backwards. It shows the character of the fracture of the fibula in this form of injury in a typical manner. It also illustrates very well the fact that a radiogram taken in this plane does not show the very considerable backward and upward displacement of the tibial fragment which almost always exists and which was most marked in this particular case. The omission to take radiograms of fractures of the ankle in two planes crossing each other at right angles has, in my experience, frequently led to disastrous results. The skiagram is taken at an angle which does not show the really great displacement which existed.

the fracture of the fibula. Fig. 2 shows the condition of the part after recovery.

Now in either of the above conditions if by any process of manipulation and fixation in splints $I$ am able to retain the fragments in apposition I am satisfied to deal with them in

EIG.2.

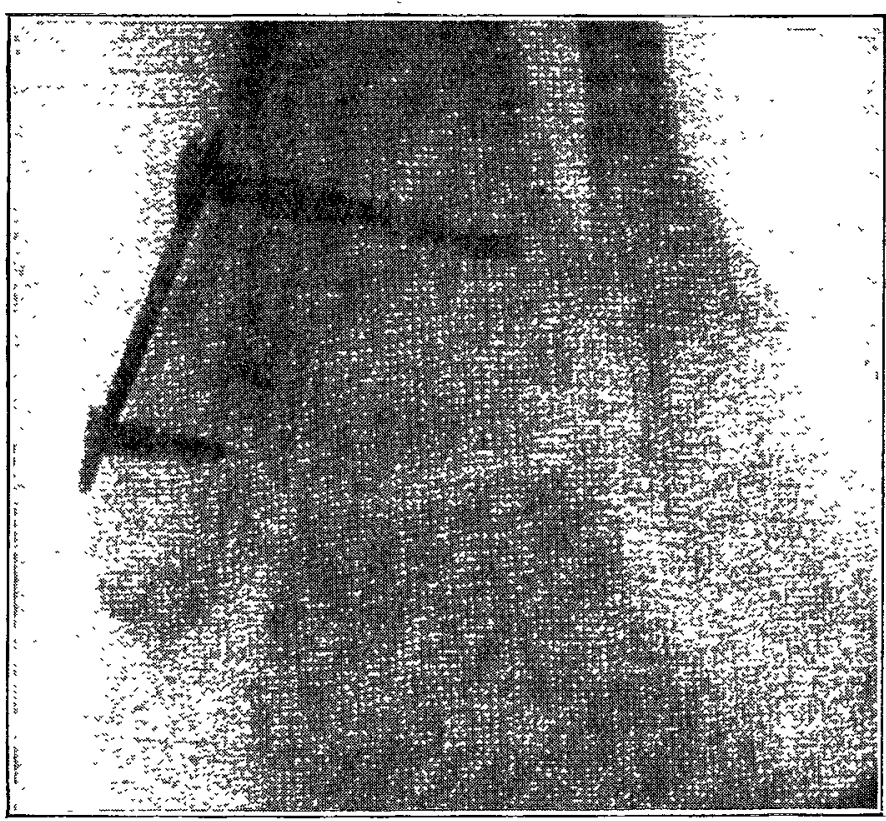

Shows the condition of the parts after the patient had re sumed his usual habits of life. The steel plate is seen lving on the inner aspect of the malleolus and shaft of the tibia, to both of which it is securely fixed by screws of suitable lengths.

that manner. In a very alcoholic patient or in a fat woman I prefer to deal with them in this way even if I cannot effect anything approaching perfect apposition, for the reason that the lower end of the tibia and fibuia in such people is very friable, the compact tissue being very thin.
This produces a comminution or scrunching of the fragments, rendering accurate apposition by operation difficult or impossible because of the loss of substance. The retention of the fragments in position often cannot be effected, for whether screws, wires, plates, or staples are used their hold upon the bone is insufficient to be of any service.

FIG. 3.

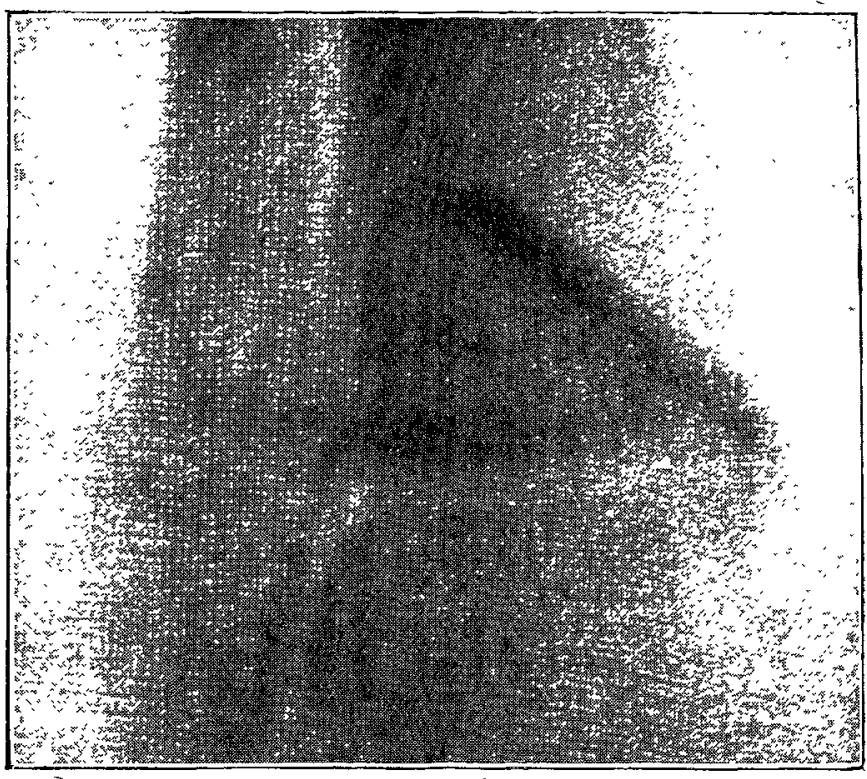

Represents an "adduction fracture" of the ankle in which the tibial fragment was secured by means of a single long screw. As in Fig. 2 the external malleolus is restored to its normal relationship to the astragalus, its fracture requiring no further operative treatment for its fixation.

In the ordinary subject, when I am able to satisfy myself that apposition cannot be effected by manipulation, I do not hesitate to advise operative interference. How cases in each of these two groups must be treated by operation depends largely on the details of the injury. Roughly speaking, in an abduction or Pott's fracture, since in these circumstances the fracture in the fibula is almost always clean and the texture of the bone firm, I prefer to expose it and to unite the frag. ments in perfect apposition by silver wire perforating the fragments, by metal plates with screws, with screws alone, and very occasionally with staples. Very frequently $I_{n}$ find $a$

FIG. 4.

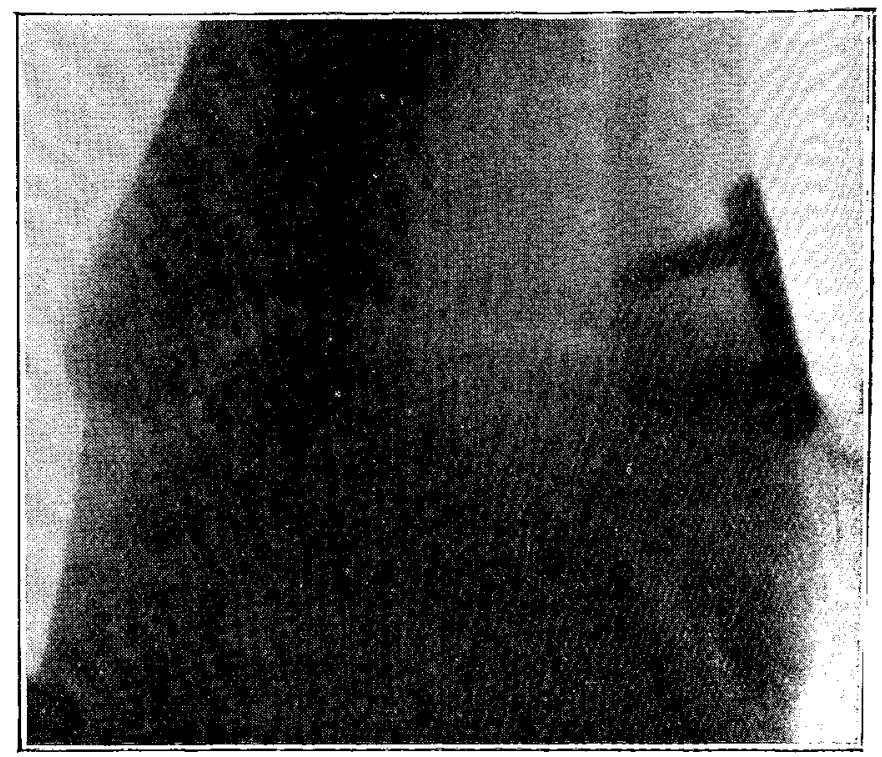

Represents an "abduction fracture," or Pott's fracture, of the ankle in which the displaced fibular fragment has after replacement in position been secured immoreably by a plate and screws.

combination of these methods very useful : for instance, the fragments may be perforated and a wire introduced which will include in its grip a plate through which screw = are criren into the malleolus and into the fibula abore the fracture. In this way not only are the surfaces retained in apposition 
by means of the encircling wire but a very stout steel splint is supplied which takes almost all the strain off the junction and absolutely prevents any buckling of the fragments on one another. On effecting accurate apposition of the fibular fragments it is usually found that in the fracture of the back of the tibia and in that of the internal malleolus, should either or both exist, these fragments come into their normal position, but should the internal malleolus appear to be at all mobile I cut down on it and fasten it with wire, plate and screws, staple, or by a long screw perforating the inner malleolus and shaft obliquely in an outward and upward direction. Fig. 3 shows the manner in which the inner malleolus may sometimes be fixed with the greatest advantage by means of a single screw, the fibular fragments coming into accurate apposition. Fig. 4 represents a Pott's fracture in which the inner malleolus was not broken. The fibular fragments were secured in perfect apposition by means of a steel plate and screws. Fig. 5 is the result of an operation for a very bad Pott's fracture, accompanied by very considerable displacement of the foot and by a fracture of the internal malleolus and back of the tibia. The tibial fractures are not well shown in this illustration, the

FIG. 5 .

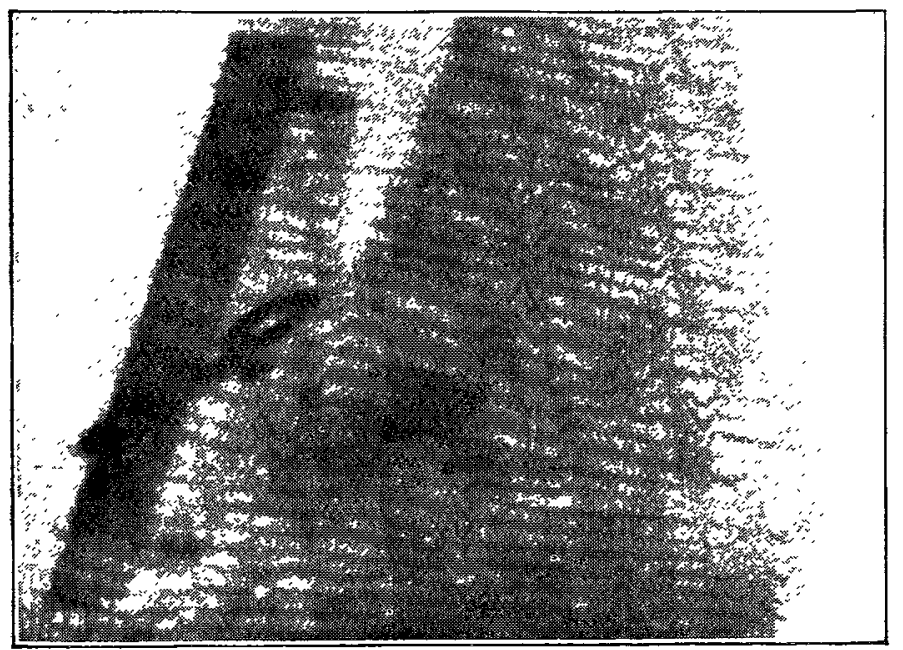

Represents the result of a bad case of abduction fracture in which the inner malleolus and the fibula were broken with a large piece of the back and outer part of the lower end of the tibia. The dislocation and replacement of fragments were effected with great difficulty. A long steel plate with screws at either end, assisted by a stout silver wire, which encircled the seat of fracture and the plate, afforded a means of effecting securely the apposition of all the fragments.

fragments having been replaced in accurate apposition. The fibular junction has been secured by a steel plate, two screws, and an encircling wire.

The knee-joint, except for fracture of the patella, which calls for no special remarks, rarely sustains fracture of the ends of the femur or tibia. In separation of the epiphysis of the femur I have found it necessary to expose the seat of fracture in order to replace the fragments in position. As a rule there is little tendency to displacement, but if there is a couple of staples afford the most effective means of securing the surfaces in apposition. In vertical fractures into the joint I have found screws or wire efficient.

In fractures about the hip-joint, as in the shaft of the femur, long steel plates with several screws perforating both layers of compact tissue and placed at suitable angles have served best to retain the fragments in accurate apposition. Fig. 6 represents a transverse fracture of the femur through the upper part of the lesser trochanter. 'There was some comminution of the upper fragment, a triangular fragment of bone being driven into the medulla. Every effort to replace the fragments by manipulation failed because of the displacement outwards and upwards of the upper fragment. The fragments were exposed, the depressed piece of bone was levered into position, the broken surfaces were brought into accurate apposition and were retained securely in position by stout steel plates and screws. That the junction effected was secure was shown by the fact that though the man during the momentary absence of the nurse flung himself out of bed and subsequently developed delirium tremens, and though his splint had to be removed, nevertheless the fragments remained in accurate apposition, as is

shown by Fig. 7, which was taken when the man left the hospital.

In extension and abduction fractures of the wrist, commonly called Colles's fractures, if the fragments cannot be replaced by manipulation I make a vertical incision over the posterior and outer aspect of the seat of fracture and

FIG. 6.

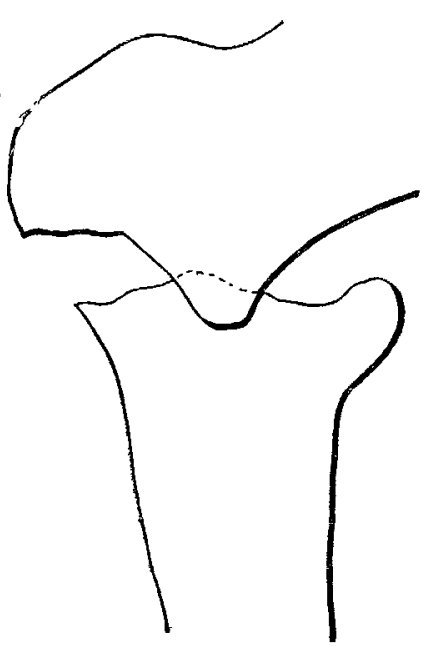

Fig. 6 represents the tracing of a radiogram of a fracture of the femur about the level of the lesser trochanter; and Fig. 7 shows a tracing after operation. These outlines were substituted for the original radiograms as these were not sufficiently clear for reproduction. The plates and screws which retained the fragments in position are also depicted.

introducing a steel elevator beneath the lower fragment, which may be formed by the epiphysis, lever it into position. This can only be done when the lower fragment is not comminuted. The hand is then fixed in a position of complete flexion and abduction. It is but rarely necessary to introduce any metal, since once placed accurately in position the fragment does not tend to become displaced.

Fractures of the olecranon are, I believe, most effectually dealt with by means of a long screw. Occasionally the screw may require removal after a long interval, because of the

FIG. 8.

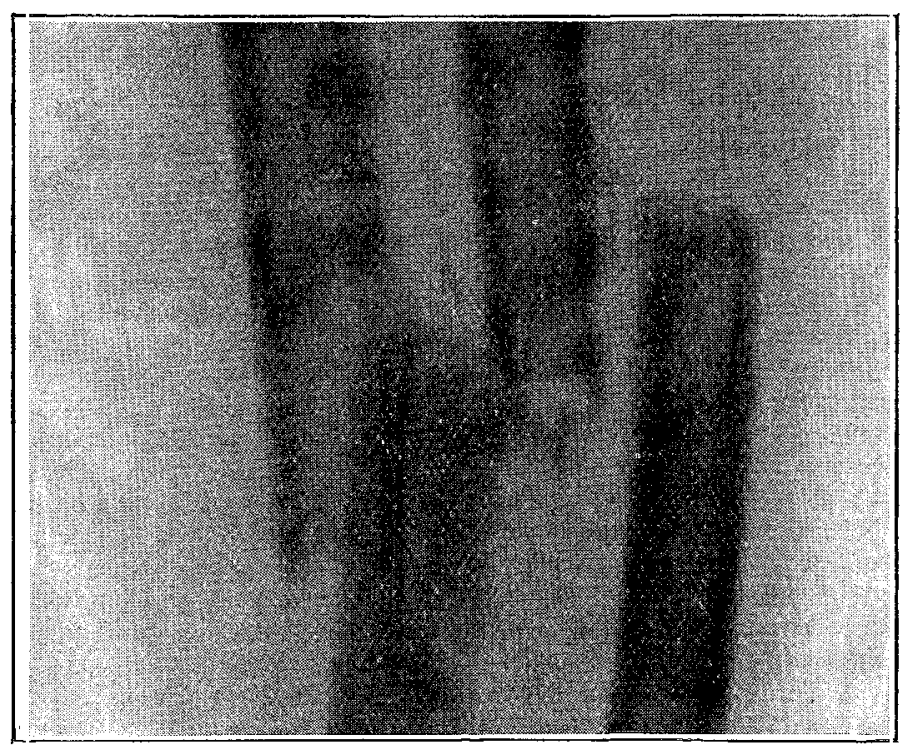

Represents an ununited fracture of the radius and ulna.

gradual exposure of its head. I have removed one 11 years after the operation for this reason. Fractures of the coronoid process are sometimes very difficult to deal with. On the whole, I believe that excision of the fragment is generally the best treatment. Much depends, however, on the nature of the fracture and the displacement of the fragment.

Fractures of the head of the radius are sometimes the cause of some anxiety as to the best measures that should be adopted. Sometimes I have replaced the fragment, 
fastening it in position. The difficulty $I$ experienced was that owing to the small size of the fragment and its friability I was unable to fix it sufficiently firmly to permit of rotatory movements being commenced at once and while waiting for union adhesions were liable to form and limit movement. Again, in some cases $I$ have removed the fragment and in others removed the stump of the head as well. In both very satisfactory results have ensued. If by manipulation the fragment can be restored to, or nearly to, its normal position passive movements undertaken at once form the best treatment.

The fracture about the elbow-joint that more frequently calls for operative treatment is that commonly called separation of the lower epiphysis of the humerus. In a small proportion of cases I have found it impossible to replace the epiphysis in perfect position by manipulation, but I have done it by making a vertical incision over the posterior aspect of the fracture, and by introducing a steel point between the fragments I have supplemented the leverage action exerted upon the fracture by the radius and ulna and so managed to restore the bone to its original form and usefalness. I have ceased to use any retaining media in these circumstances, since there is no tendency to displace. ment if the elbow is kept fully flexed. If the fragment is displaced laterally as well as backwards the lateral dis. placement must be reduced before that in an antero-posterior plane.

FIG. 9.

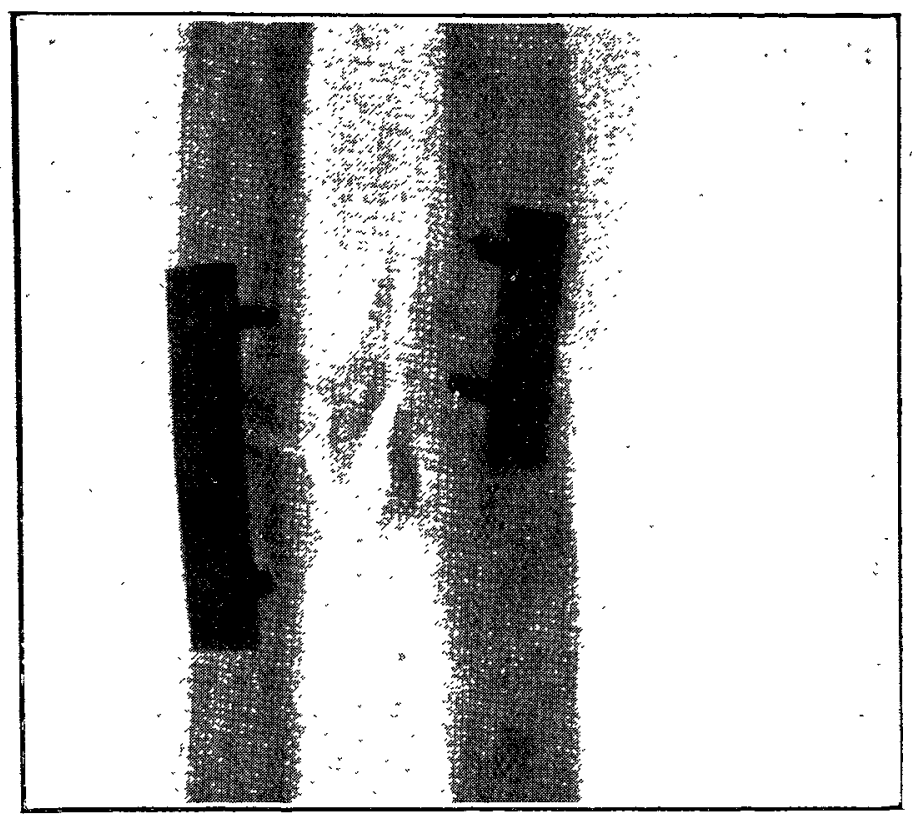

Shows the results of operation on Fig. 8 , the replaced frag. ments having baen seaured by screws and plates.

The last joint to which I will call attention is the shoulder. In fractures of the upper end of the humerus in which I have been unable by manipulation to restore the bone to its original form and in which it was important that the functions of the joint should be perfect I have exposed the fracture and replaced the fragments in position. In many cases, as in separation of the epiphysis and in transverse fracture of the surgical neck, once accurate apposition has been obtained there is no tendency to displacement, but if the fracture is oblique or if the bones are comminuted some retaining medium must be employed. Staples are very useful when it is necessary to perforate the trochanters because of their cancellous structure, but if the fragments offer a dense hold screws or screws and plates are very useful. The results of such operations are as good as possible.

Now a few words as to the best media for retaining fragments in accurate apposition. I find screws in two gauges most useful ; up to three-quarters of an inch Gauge 5 is as good as any. As three-quarters of an inch is a very useful size I use it in Gauge 7 also, which is the gauge of all the screws over that length. Having only two gauges three diamond drills only are required, one for the thread of the screw of Gauge 5 , one for the barrel of Gauge 5 and for the thread of Gauge 7, and one for the barrel of Gauge 7. Stout metal plates can be usefully combined with the screws. They are made in various lengths with two, three, or more holes. Some of the plates are of steel, while others which are of
FIG. 10.

$\stackrel{a}{1}$

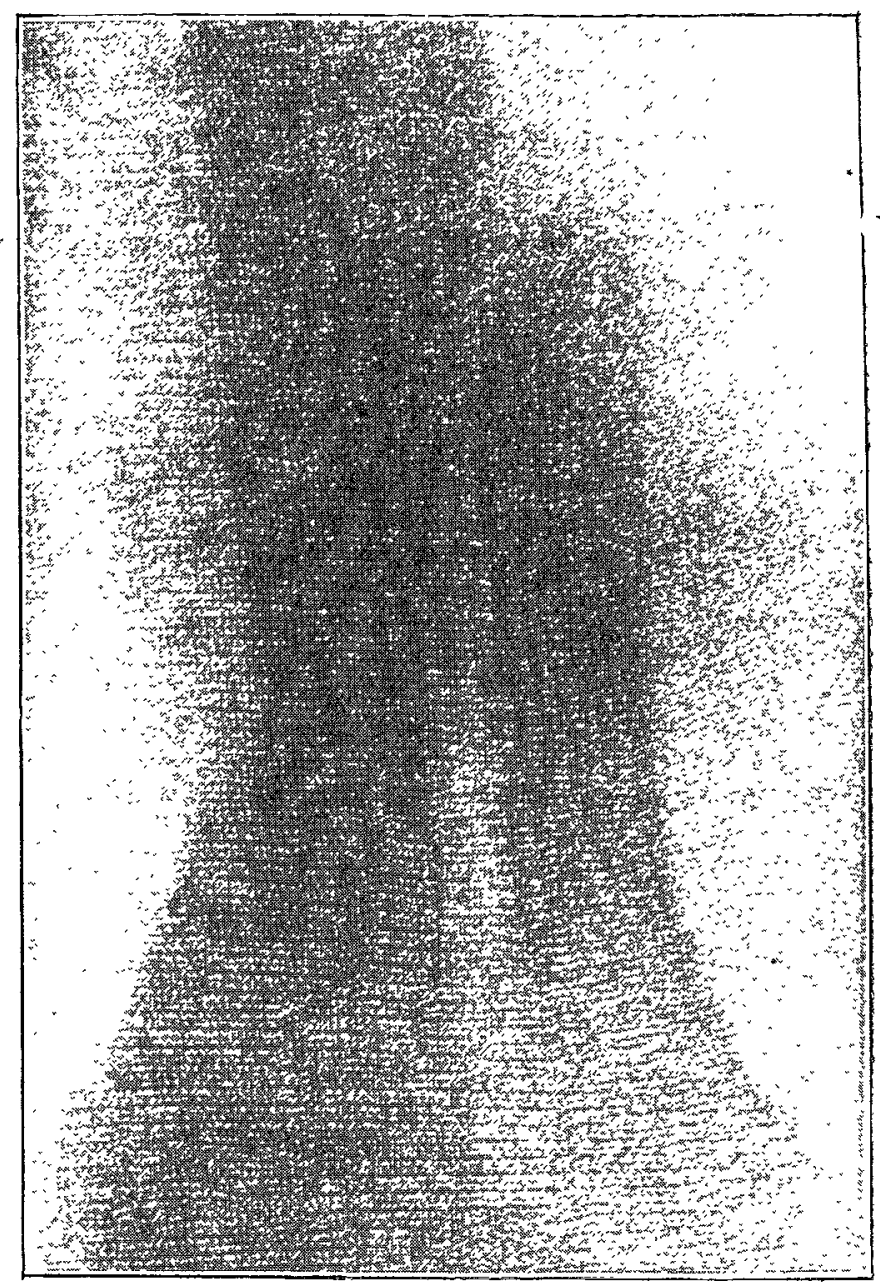

Illustrates a comminuted fracture of the lower end of the femur in which the fragments were much displaced.

FIG. 11.

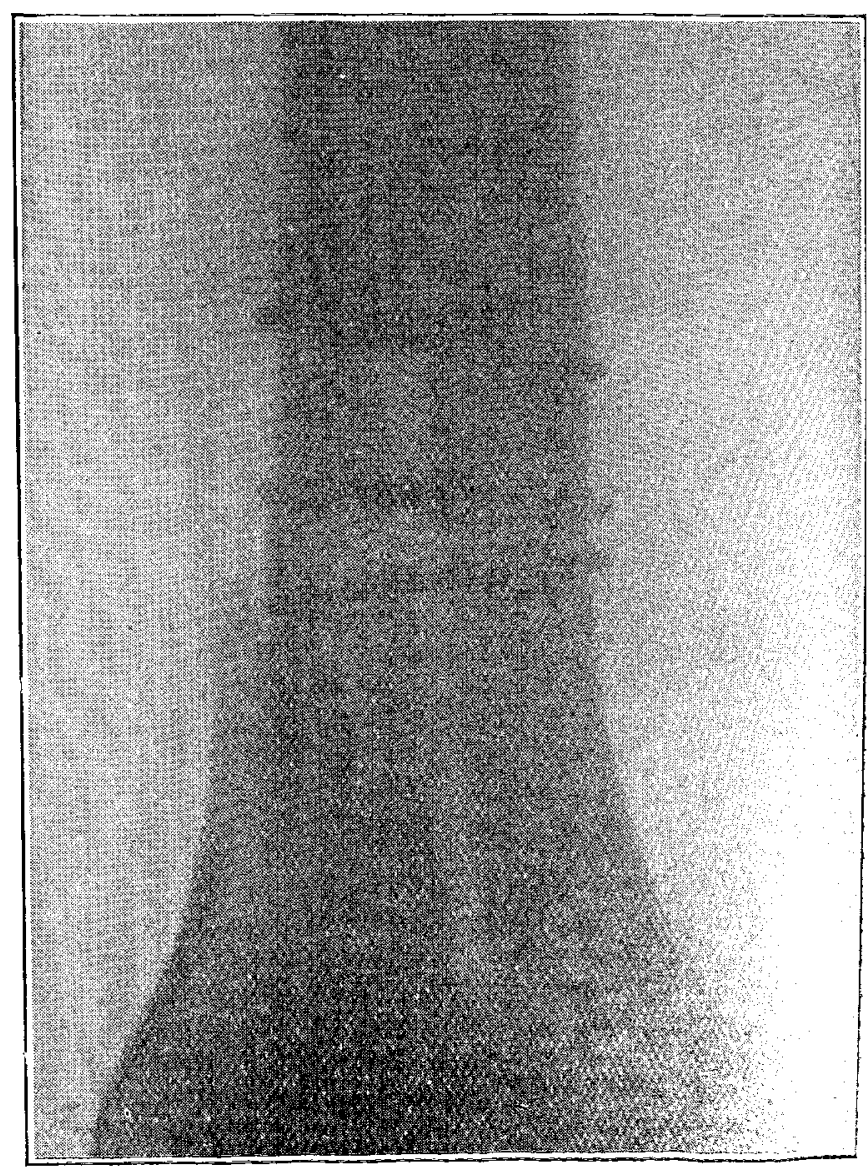

Show's the result of operation on the fracture illustaic.: in Fig. 10. 
hard German silver can be best employed to fit the curves of bones. Those used for fractures of small bones like the radius, ulna, clavicle, and fibula are drilled to the 5 Gauge which allows the passage of a screw that will perforate both compact layers, a matter of vital importance since a screw obviously cannot get a secure hold on a single compact layer. To illustrate this I have chosen Fig. 8 which represents an ununited fracture of nearly three months' standing rather than a recent fracture, since the difficulties to be encountered are much greater in the former than in the latter. The injury was sustained on Feb. 22nd, 1906, and came under the care of a surgeon who has devoted much attention to the mode of production of fractures. I mention this fact to show that the patient had the advantage of the best treatment possible at the time of the injury. Manipulation was effected under an anæsthetic and plaster-of-Paris was employed for fixation. A useful arm was promised in about six weeks. On May 17th, we exposed the fragments, removed their ends, and effected accurate apposition by screws and plates. The patient has now a perfectly useful arm. Fig. 9 represents the fragments united by means of the plates and screws.

Fig. 10 represents a more or less vertical fracture of the lower end of the femur into the knee-joint in which the condyles were separated by a considerable interval. Fig. 11 shows the condition after operation.

Virgin silver appears to afford one of the best wire melia should such be required. Staples with prominent or rounded angles are often very efficient in conditions suitable to their use, as in the case of the upper end of the humerus and the lower end of the femur. I know no condition in which pegs can be of any use mechanically.

Cavendish-square, $\mathrm{W}$.

\section{THE OPSONIC INDEX TO VARIOUS ORGANISMS IN THE SANE AND INSANE,}

WITH THE RESULTS PRODUCED BY INJECTING TUBERCULIN. ${ }^{1}$

By O. J. SHAW, M.D. EdIN., M.R.C.P. EDIN.,

ASSISTANT MEDICAX OFFICRR, ROYAL ASYLUM, MONTROSE, N.B.

THE recent observations of Wright and others on the opsonic power of human blood serum to the tubercle bacillus and other organisms and the methods they have discovered of estimating this power have opened up a new field for research and provided new methods of treatment for bacterial diseases which have already produced beneficial results. As Wright ${ }^{2}$ found that in cases of staphylococcal and tuberculous infection with an entire absence of clinical symptoms there was a diminished phagocytic power to these organisms as compared with that of healthy individuals, and as this power could be increased by injections of the corre sponding vaccine, he concluded that the lowered phagocytic power permitted infection to occur and that the resistance to infection by any organism could be estimated by calculating the opsonic power of the blood serum. The results of observations recorded by Bulloch and others agree with those obtained by Wright and support his view, as all have found a lowered opsonic power in cases of localised tubercular and staphylococcal infection, and these are the cases which benefit most from vaccine injections. Low tuberculo opsonic indices have also been found in cases of early phthisis "cured" by sanatorium treatment. These cases frequently relapse, however, and whether the low opsonic index is the cause or the result of infection is not definitely decided. The estimation of the opsonic index of a class of healthy patients with a decided liability to tuberculous infection is therefore an important point in estimating the value of a low index as an indication of the resistive power of the individual to infection.

The statistics of the death-rate from tubercular disease, particularly phthisis, occurring in asylums leave no room for doubt that the insane as a class are specially liable to taberculous infection. In their last report the English Commissioners in Lunacy compare the death-rates in institutions for the insane with those of the general population, all deatbs under the age of 15 years being excluded. The death-rate of the general population per 1000 from phthisis was for males

1 Extract from a thesis for the degree of M.D. Edinburgh University 2 THE LanceT, Dec. 2nd, 1905, p. 1598.
$2 \cdot 07$ and for females $1 \cdot 35$, while in the insane population th was $16 \cdot 0$ and $14 \cdot 6$ for each sex respectively. The Scottish Commissioners in their report for 1905 refer to the same subject and quote figures which are equally striking. In the six district asylums showing the lowest death-rate from tuberculous disease, of which Perth district asylum is one, the average per 1000 patients resident was 11 , while in the counties from which these asylums draw their patients the average per 1000 persons living between the ages of 20 and 60 years was $2 \cdot 8$. Clouston says that in asylums consumption is between three and four times more common than in the general population at the same ages, and the figures quoted above more than support his statement. Even although a certain amount of the difference in the death-rates might be accounted for by overcrowding or other unfavourable conditions in some asylums or by the greater number of post-mortem examinations, and more complete and accurate returns made by institutions, or by the great difference in the totals from which the averages are estimated, still the fact remains that the insane are more susceptible to tuberculosis than ordinary individuals.

I have made observations on the opsonic indices of six members of the staff of Perth District Asylum and also on 30 patients suffering from various forms of mental disease. All were at the time of observation free from active tuberculous disease but five of the patients examined showed evidences of having at one time suffered from such infection. Of these cases one had cicatrices in the neck, while the others showed signs of former lung mischief. Two of them were suffering from general paralysis, one of whom has since died, and only a few small cicatrices at the apex of one lung were found post mortem. The usual technique for the estimation of the opsonic index was employed, equal quantities of serum, leucocytes, and bacterial suspension being used. The blood for examination was taken at the same hour each day and five consecutive observations were made in each case. Koch's new tuberculin T.R. was then injected and daily observations were made for a further period. My own leucocytes were used throughout and my blood serum was used as control. Its opsonic power was fairly constant, the number of bacteria ingested showing little variation from day to day. For the estimation of the tuberculo-opsonic index the contents of 80 leucocytes were usually counted and in no case were less than 50 enumérated. Simultaneous observations were made on the opsonic power of various cases to the bacillus coli communis, staphylococcus aureus, and micrococcus rheumaticus. These observations were continued after the injection of tuberculin T.R. The contents of 50 leucocytes were usually counted for these observations, but on a few occasions only 30 were examined. The same number was examined in each case on the same day.

The average tuberculo-index of the six healthy control cases for five consecutive days was $1 \cdot 07$, the average for each case being $0.98,0 \cdot 97,1 \cdot 32,1 \cdot 06,1 \cdot 01$, and $1 \cdot 11$, showing a variation between $0 \cdot 97$ and $1 \cdot 32$. This average index agrees with the results obtained by other observers in healthy persons. Urwick ${ }^{3}$ in 20 cases, found the average index to be 1006 , while Bulloch ${ }^{4}$ in 84 cases found an index of 0.96 , and in Lawson and Stewart's ${ }^{5} 25$ cases the average was 1. The total variation, $0 \cdot 35$ is less than that found by Bulloch in the 84 cases he examined. Urwick states that there is little variation in the index of a healthy person from day to day. I found considerable daily variation in some cases, though the observations were made as far as possible under the same conditions. The greatest amount of variation recorded between any two consecutive observations was $0 \cdot 3$. In one control case, however, a record so low as 0.68 was obtained on one occasion.

The average tuberculo-opsonic index of the 30 insane persons examined for five days was $0 \cdot 88$. The variation was between 0.65 and 1.23 and the daily fluctuation was greater than in the healthy control cases. Of these 30 cases the six with the highest indices gave an average of 1.07 , which is exactly that found in the same number of control cases. Although a greater number of insane were examined it may be fairly concluded that the average tuberculo-opsonic index of the non-tubercular insane is below that of the healthy sane. The average of the five patients with evidences of old tubercular disease was the same as that found for all the 30 cases. 3 Brit. Med. Jour, July 22nd, 1905. 4 London IIospital Gazette. vol. xi., March, 1905. The Lancet, Dec. 9th, 1905, p. 1679. 